\title{
Nonprofessional Phagocytosis Can Facilitate Herpesvirus Entry into Ocular Cells
}

\author{
Vaibhav Tiwari ${ }^{1}$ and Deepak Shukla ${ }^{2,3}$ \\ ${ }^{1}$ Department of Microbiology and Immunology, Midwestern University, Downers Grove, IL 60515, USA \\ ${ }^{2}$ Department of Microbiology and Immunology, University of Illinois at Chicago, \\ 1855 W. Taylor Street, LIERI Building, Chicago, IL 60612, USA \\ ${ }^{3}$ Department of Ophthalmology and Visual Sciences, University of Illinois at Chicago, \\ 1855 W. Taylor Street, LIERI Building, Chicago, IL 60612, USA
}

Correspondence should be addressed to Deepak Shukla,dshukla@uic.edu

Received 12 November 2011; Accepted 13 December 2011

Academic Editor: Aziz Alami Chentoufi

Copyright (C) 2012 V. Tiwari and D. Shukla. This is an open access article distributed under the Creative Commons Attribution License, which permits unrestricted use, distribution, and reproduction in any medium, provided the original work is properly cited.

\begin{abstract}
Phagocytosis is a major mechanism by which the mediators of innate immunity thwart microbial infections. Here we demonstrate that human herpesviruses may have evolved a common mechanism to exploit a phagocytosis-like entrapment to gain entry into ocular cells. While herpes simplex virus-1 (HSV-1) causes corneal keratitis, cytomegalovirus (CMV) is associated with retinitis in immunocompromised individuals. A third herpesvirus, human herpesvirus-8 (HHV-8), is crucial for the pathogenesis of Kaposi's sarcoma, a common AIDS-related tumor of eyelid and conjunctiva. Using laser scanning confocal microscopy, we show that successful infection of ocular cell types by all the three viruses, belonging to three divergent subfamilies of herpesviruses, is facilitated by induction of F-actin rich membrane protrusions. Inhibitors of F-actin polymerization and membrane protrusion formation, cytochalasin D and latrunculin B, were able to block infection by all three viruses. Similar inhibition was seen by blocking phosphoinositide 3 kinase signaling, which is required for microbial phagocytosis. Transmission electron microscopy data using human corneal fibroblasts for HSV-1, human retinal pigment epithelial cells for CMV, and human conjunctival epithelial cells for HHV-8 are consistent with the possibility that pseudopod-like membrane protrusions facilitate virus uptake by the ocular cells. Our findings suggest a novel mechanism by which the nonprofessional mediators of phagocytosis can be infected by human herpesviruses.
\end{abstract}

\section{Introduction}

Phagocytosis is essentially a form of endocytosis wherein particles are trapped and enclosed by cell membrane protrusions. Our knowledge of phagocytosis comes mainly from professional phagocytes such as macrophages and neutrophils, which fight against microbial invasion and removal of dead cells [1]. However, in many cases, nonprofessional phagocytes including epithelial cells and fibroblasts of ocular origin have also been shown to possess the ability to phagocytose their adjacent apoptotic cells or spent cell debris [1-3]. Well-known examples include Sertoli cells in testis [3] and the retinal pigment epithelial (RPE) cells in the retina [3]. Recently we demonstrated that herpes simplex virus-1 (HSV-1) has the ability to exploit phagocytosis to promote its entry into corneal fibroblasts [4]. Similar findings have been made with amoebal mimivirus [5, 6]. Nonprofessional phagocytosis is also triggered by the recognition of ligands by corresponding receptors on phagocytosing cells. This results in surrounding of the target particles with a specialized pseudopod-like extension of the plasma membrane. The local reorganization of F-actin underneath the extension and the contractile motors supporting the reorganization provide the driving forces for trapping the particles $[2,7$, 8]. Similar to professional phagocytosis by macrophages and neutrophils, nonprofessional phagocytosis also requires phosphoinositide 3 kinase (PI3K) signaling [6].

Herpesviruses are highly prevalent among humans [9]. A vast majority of adult human population is seropositive for multiple herpesviruses, which cause life-long infections and virtually all are capable of causing ocular manifestations $[9,10]$. The family of herpesviruses, which may have more 
than a hundred known members, has been divided into three subfamilies. Among human herpesviruses, alphaherpesvirus subfamily is exemplified by herpes simplex virus-1 (HSV1 ), betaherpesvirus subfamily by cytomegalovirus (CMV), and gammaherpesvirus subfamily by human herpesvirus- 8 (HHV-8) [9]. The most common eye infections are caused by HSV-1, which is a well-studied cause for herpes stromal keratitis (HSK), a blinding eye disease. In addition HSK is also associated with blepharitis, dendritic keratitis, disciform stromal edema, and conjunctivitis [11]. The involvement of CMV and HHV-8 in ocular diseases is mostly limited to immunocompromised human population which includes AIDS patients and organ transplant recipients [10]. CMV used to cause retinitis in a significant number (30\% or more) of AIDS patients. Lately, this situation has been brought under control by introduction of highly active antiretroviral therapy (HAART). However, the ocular problems associated with HHV-8 remain very common among the AIDS patients who often suffer from the tumors of eyelid and conjunctiva [12].

The mechanisms by which herpesviruses enter into host cells vary with individual viruses [4, 13-15]. For instance, all the three herpesviruses discussed previously use separate entry receptors, prefer certain cell types over others for infection and the establishment of latency, and use different mode(s) of entry [16]. In the case of HSV-1, endocytosis and nonprofessional phagocytosis play a dominant role in infection of many cell types $[4,14,17]$. Recent studies have indicated that HSV-1 entry may be atypical endocytosis since it is not mediated by formation of clathrin-coated pits or caveolae and it may or may not be $\mathrm{pH}$-dependent $[18,19]$. CMV and HHV-8, in contrast, may enter into cells by clathrin-coated endocytic cup formation and the entry is $\mathrm{pH}$ dependent [15]. While the significance of endocytosis may be known, it is not clear how herpesviruses infect cells of ocular origin, many of which are immune privileged [20]. It is also unknown if actin cytoskeleton plays a direct role in the initiation of infection, and likewise, the significance of pseudopod-like protrusions in entry process has not been described for herpesviruses. Here we demonstrate a unique commonality in terms of entry mechanism among three representative members of herpesvirus subfamilies. We show that the entry of the viruses into target cell types of ocular origin is facilitated by F-actin containing pseudopodlike membrane protrusions. Similar to phagocytosis, this entry mechanism is also controlled by PI3K signaling. The protrusions formed during nonprofessional phagocytosis may facilitate viral uptake since inhibition of protrusion formation significantly inhibits viral entry or uptake into cells. Our findings also demonstrate a possible way by which herpesviruses may have evolved to escape from neutralization by the innate mediators of phagocytosis.

\section{Materials and Methods}

2.1. Cell Cultures. Retinal pigmental epithelial (RPE) cells were grown in Dulbecco's modified Eagle's (DMEM) medium supplemented with $10 \%$ fetal bovine serum (FBS) containing penicillin and streptomycin [21]. Primary cultures of human corneal fibroblasts (CFs) cells were grown in DMEM medium containing $10 \%$ FBS and 5\% calf serum (CS) as previously described [22]. Primary cultures of human conjunctival epithelial (HCE) cells were kindly provided by Dr. Ilene K. Gipson (Schepens Eye Research Institute; Harvard Medical School). HCE cells were cultured on GIBCO keratinocytes serum-free medium with supplied BPE (bovine pituitary extract) and EGF (0.2 ng/mL) [23]. CMV virus was detected by a monoclonal antibody specific for glycoprotein B (Virostat Inc., Portland, ME). HHV-8 virus was detected by a monoclonal antibody specific for HHV-8 envelope glycoprotein K8.1A and FITC-conjugated secondary antibody.

2.2. Viruses. The $\beta$-galactosidase-expressing recombinant HSV-1 gL86, GFP-expressing HSV-1 (K26GFP-HSV-1), cytomegalovirus (CMV; Towne strain RC256), and HHV-8 virus (wild-type and rKSHV.152) were used in this study. HSV-1 gL86 was propagated in gL complementing cell line (79B4 cells). GFP-expressing HSV-1 (K26GFP) virus was grown in Vero cells [4]. The viruses were purified by sucrose density gradient as previously described [22].

2.3. Electron Microscopy. CF, RPE, and HCE cells cultured in Lab-Tek chamber slides and in Anopore wells (Nalge Nunc) (approximately $1.5 \times 10^{5}$ cells/well) were infected with purified HSV-1, CMV, and HHV-8 at 50-100 MOI for 90-120 min as previously described [4]. Infected cells were rinsed with serum-free buffer and fixed with 1\% osmium tetroxide for $10 \mathrm{~s}$, immediately followed by the aldehyde fixation for $1 \mathrm{hr}$ (2.5\% glutaraldehyde, $2 \%$ paraformaldehyde in $100 \mathrm{mM}$ cacodylate buffer, $\mathrm{pH}$ 7.4). Cells were rinsed three times for $5 \mathrm{~min}$ with $100 \mathrm{mM}$ cacodylate buffer, postfixed for $1 \mathrm{hr}$ in $1 \%$ osmium tetroxide, stained with uranyl acetate, dehydrated through a graded ethanol series, and finally embedded using Embed. For transmission electron microscopy (TEM) (JEM-1220; JEOL USA Inc.) images were captured at $1,000-600,000 \mathrm{x}$, point- $0.36 \mathrm{~nm}(3.6 \mathrm{~A})$, and lattice- $0.2 \mathrm{~nm}(2 \mathrm{~A})$ at room temperature, ACC voltage 40$120 \mathrm{kV}$ using a Gatan camera (Digital CCD; Gatan Inc.), and Gatan Digital Micrograph (DM) v2.5 acquisition software.

2.4. Indirect Immunofluorescence. Target CF, RPE, and HCE cells cultured in Lab-Tek chamber slide were infected with herpes viruses for 90-120 minutes. The cells were then stained. For cell surface immunofluorescence, the cells were incubated with primary antibodies at $4^{\circ} \mathrm{C}$ for $45 \mathrm{~min}$, washed 10 times with cold PBS, and fixed with acetone for $10 \mathrm{~min}$ at $-20^{\circ} \mathrm{C}$. $\mathrm{HHV}-8$ and $\mathrm{CMV}$ viruses were detected by anti-gB (CMV) and anti-K8.1A (HHV-8) antibodies and FITC-conjugated secondary antibodies. Actin fibers were also stained with $10 \mathrm{nM}$ rhodamine-conjugated phalloidin (Molecular Probes, Carlsbad, California, USA) to compare the number of actin-rich protrusion produced in the infected cells compared to uninfected cell. The cells were washed before mounting in Vectashield mounting medium (Vector Laboratories, Inc. Burlingame, CA). Leica confocal microscope SP2 was used for imaging. 
2.5. Viral Entry Assay. Viral entry assay was based on quantitation of $\beta$-galactosidase expressed from HSV-1 genome by soluble $\mathrm{O}$-nitro-phenyl- $\beta$-D-galactopyranoside (ONPG, Pierce Biotechnology), $3 \mathrm{mg} / \mathrm{mL}$. Similarly CMV $\beta$-galactosidase-expressed reporter virus from ATCC was used. HHV-8 virus entry was determined by fluorescence readout assay. CF, RPE, and HCE were either mock treated or treated with medium containing Cyto D $(0.5$ and $1.0 \mu \mathrm{g} / \mathrm{mL})$, Lat B $(0.25-2.5 \mu \mathrm{M})$, PI3-kinase inhibitor (LY294002), and in active compound LY303511. In parallel experiment CF, RPE, and HCE cells were transfected with PI3K dominantnegative expression plasmid $(\triangle \mathrm{iSH} 2)$ or with control plasmid followed by the viral infection [24].

\section{Results and Discussion}

We began our investigation by monitoring morphological changes that occur in ocular cells upon infection with herpesviruses. The original goal was to highlight differences in the way the herpesviruses, belonging to three distinct subfamilies, infect their target cells of ocular origin. We used primary human corneal fibroblasts (CFs), human conjunctival epithelial (HCE), and human retinal pigment epithelial (RPE) cells for infection with green fluorescent protein expressing HSV-1 (K26GFP), CMV (AD169), and HHV-8 (courtesy of J. Vieira, University of Washington, Seattle, WA), respectively. To our surprise, infection of three different cell-types by three different herpesviruses resulted in a common morphological change, which was represented by a clear enhancement in the number of Factin rich plasma membrane protrusions (Figures 1(a)$1(\mathrm{c}))$. The protrusions were $1.0-10 \mu \mathrm{m}$ large and often uniformly distributed along the surface of individual cells (Figures 1(d)-1(f)). Protrusions were observed during HSV1 invasion of CF (Figures 1(a) and 1(d)), CMV invasion of RPE cells (Figures 1(b) and 1(e)), and HHV-8 invasion of HCE cells (Figures 1(c) and 1(f)).

Since a phagocytosis-like uptake for HSV-1 has been suggested and many ocular cell-types can perform phagocytosis [4], we decided to generate ultrastructural evidence to support the possibility that projections may help with virus entrapment as well. Thus, as described previously [4], transmission electron microscopy (TEM) was performed with virally infected ocular cells (CF for HSV-1, RPE for $\mathrm{CMV}$, and HCE for HHV-8). Again, a possible significance of protrusions that were formed by extension of the plasma membrane (continuity of the plasma membrane was visible in all cases) was indicated by the presence of virus particles next to them (Figure 2). HSV-1 (Figure 2(a)), CMV (Figure 2(b)), and HHV-8 (Figure 2(c)) were all seen present inside the cups formed by plasma membrane protrusions. In many cases, protrusions seemed to entrap single virus particles (Figures 2(b) and 2(c)).

To generate biochemical evidence in support of the role of membrane protrusions in productive viral uptake, inhibitors of F-actin polymerization were examined for their ability to block the infection. The inhibitors used were cytochalasin D (Cyto D) and latrunculin B (Lat B), both block F-actin polymerization that is required for protrusion formation by the plasma membrane $[25,26]$. For HSV1 , virus uptake was determined by colorimetric analysis of entry of a $\beta$-galactosidase expressing reporter virus HSV$1(\mathrm{KOS}) \mathrm{gL} 86$. As previously described, transcription of early virus genes allows expression of $\beta$-galactosidase activity that is read by a spectrophotometer after addition of its soluble substrate ONPG [27]. A similar $\beta$-galactosidase expressionbased plaque assay was used for CMV and GFP expression in infected cells was used as a marker for virus infection of HCE cells by HHV-8 [4]. It was seen in all cases that at previously determined dosages, both inhibitors have significant negative effects on viral infection. About $50-80 \%$ HSV-1 entry into CF was blocked by the inhibitors (Figure 2(d)). Similarly, $40-75 \%$ of CMV-infected cells treated with the drugs significantly inhibited viral entry (Figure 2(e)) and 40 to $60 \%$ reduction in GFP expressing cells was observed in CytoD and Lat-B-treated HCE cells (Figure 2(f)). It is therefore likely that membrane protrusion formation is a common and important requirement for herpesvirus infection of the ocular cells.

Finally, to add more evidence in favor of the involvement of membrane protrusion formation and phagocytosis in herpesvirus uptake by ocular cells, we decided to block PI3K-mediated signaling, which is required for phagocytic cup formation [6]. As shown in Figures 3(a)-3(c), ocular cells pretreated with a PI3Kinase inhibitor (LY294002) $[28,29]$ showed decreased entry by HSV-1 (Figure 3(a)), CMV (Figure 3(b)), and HHV-8 (Figure 3(c)). A similar level of decrease was also seen when the cells were first transfected with an expression construct for a dominantnegative PI3K mutant lacking the p110-catalytic subunitbinding domain $(\triangle \mathrm{iSH} 2)$ of PI3K $[30]$ and then infected with the herpesviruses (Figures 3(a)-3(c)). In all cases it was clear that PI3K signaling was required for efficient infection by the viruses. To determine the specificity of PI3K blocking, we used a compound LY303511 (Calbiochem Inc.) that is highly related to LY294002. It was again clear that the former had no significant effect on HSV-1 (Figure 3(a)), CMV (Figure 3(b)), or HHV-8 (Figure 3(c)) entry.

In summary, we demonstrate some unique features of herpesvirus infection of the cells of ocular origin. It is evident that the ocular cells may respond to the three representative herpesvirus subfamily members by inducing F-actin-based membrane protrusions. Any blockage of protrusion formation greatly restricts viral entry (Figure 1) and inhibition of PI3K signaling, a requirement for F-actin remodeling during phagocytosis, also blocks herpesvirus entry into the ocular cells (Figure 3). PI3Ks are cellular heterodimeric enzymes that consist of a regulatory subunit (p85) activated by tyrosine phosphorylation, which recruits inositol phospholipids that are phosphorylated by the catalytic subunit (p110) [31-34]. The lipids then serve as second messengers that control phosphorylation of other kinases such as Akt/PKB, some protein kinase $C$ isoforms, cyclic AMP-dependent protein kinase A, and the ribosomal S6 kinases p70 and p85 [35]. The ability of PI3K to regulate pathways important for phagocytosis coupled with the ability of herpesviruses to modulate its activity could be an important mechanism by which the viruses exploit 


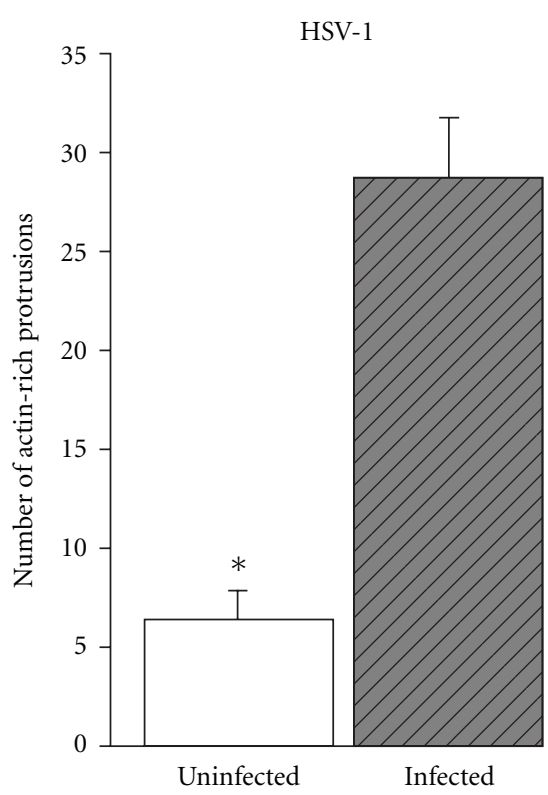

(a)

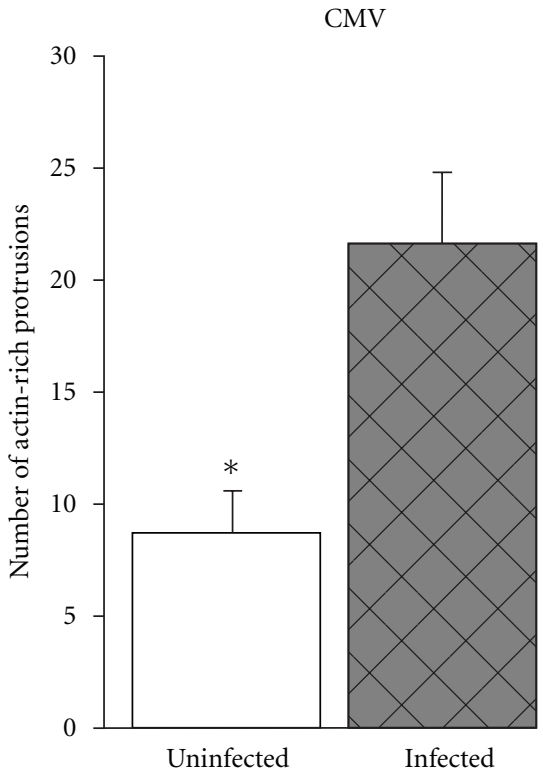

(b)
HHV-8

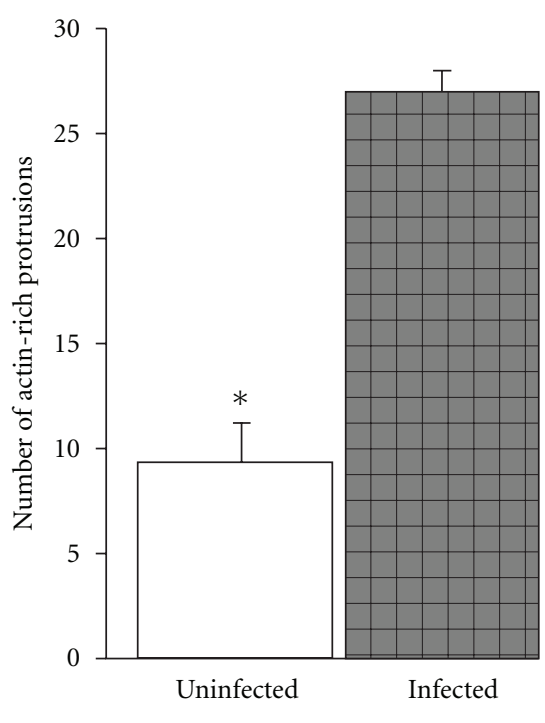

(c)
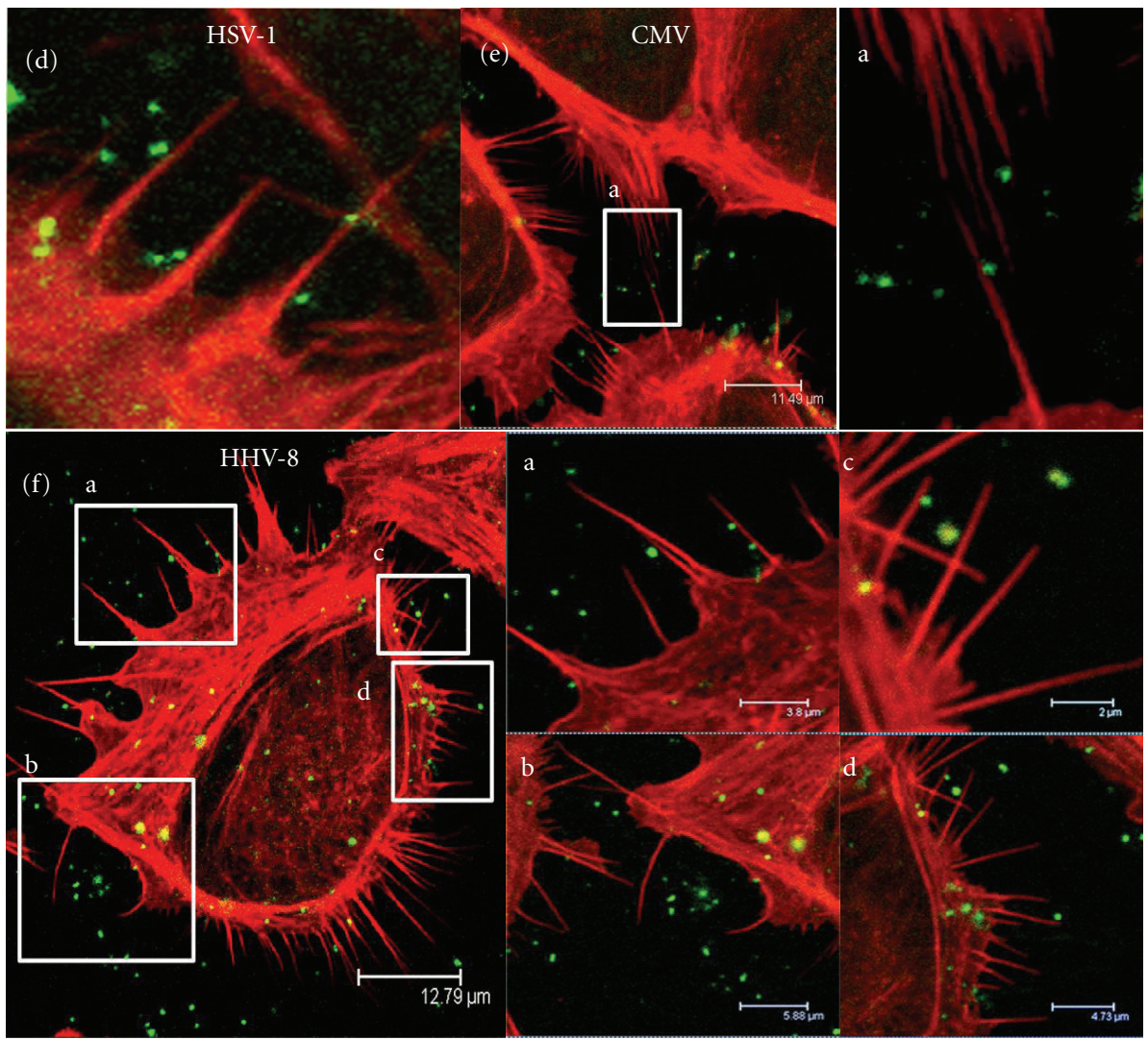

FIGURE 1: Herpesvirus infection leads to increase in the number of F-actin-rich projections. (a)-(c) Counting of the projections on per cell basis was done 15-30 min before and after the addition of the virus indicated (Herpes simplex virus type-1; (HSV-1), Cytomegalovirus; (CMV) and Human herpesvirus-8; (HHV-8)). In this and all other subsequent experiments cells used included corneal fibroblasts (CFs) (a) for HSV-1, human retinal pigment epithelial (RPE) cells for CMV (b), and primary cultures of human conjunctival epithelial (HCjE) for HHV-8 (c) infection. Four independent experiments were conducted to record the data. Numbers of actin-rich protrusions are presented as means with error bars showing standard deviation. (d)-(f) Confocal images of cells challenged with indicated viruses are shown. Virally infected cells were fixed, permeabilized, and stained for F-actin with $10 \mathrm{nM}$ rhodamine-conjugated phalloidin before mounting (Vectashield mounting medium). All confocal (Leica SP2) images were captured at 63x objective. Magnified views of the images in the boxes are shown next to them and labeled identically. CMV and HHV-8 viruses were identified by monoclonal antibodies against CMV envelope glycoprotein $\mathrm{gB}$ and HHV-8 K8.1A ( $1: 50)$ followed by secondary antibody treatment conjugated to FITC. 


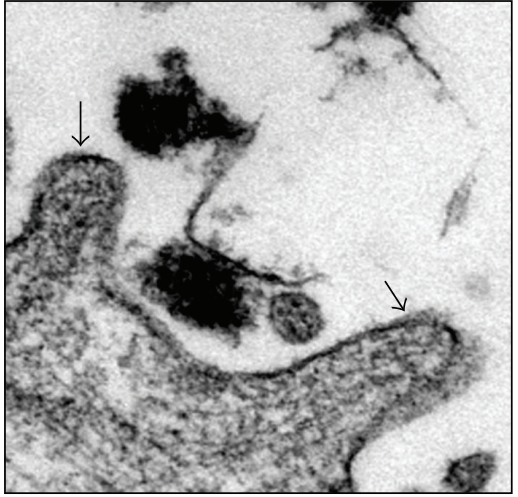

HSV-1

(a)

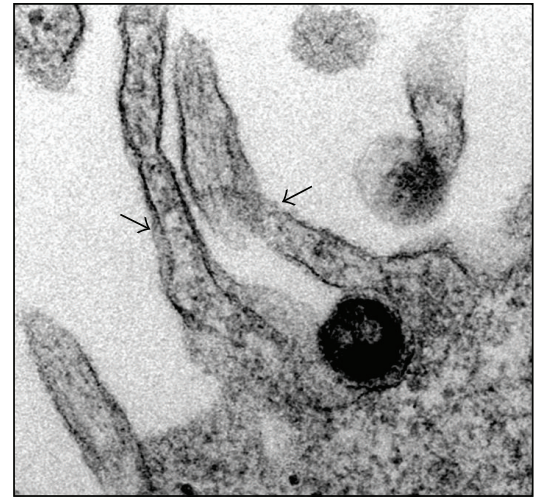

CMV

(b)

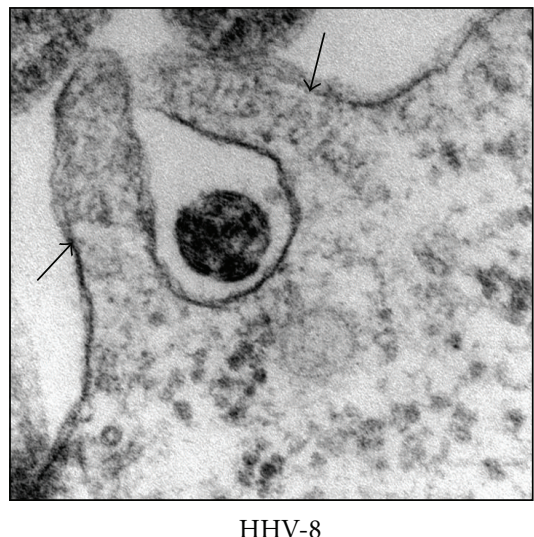

(c)

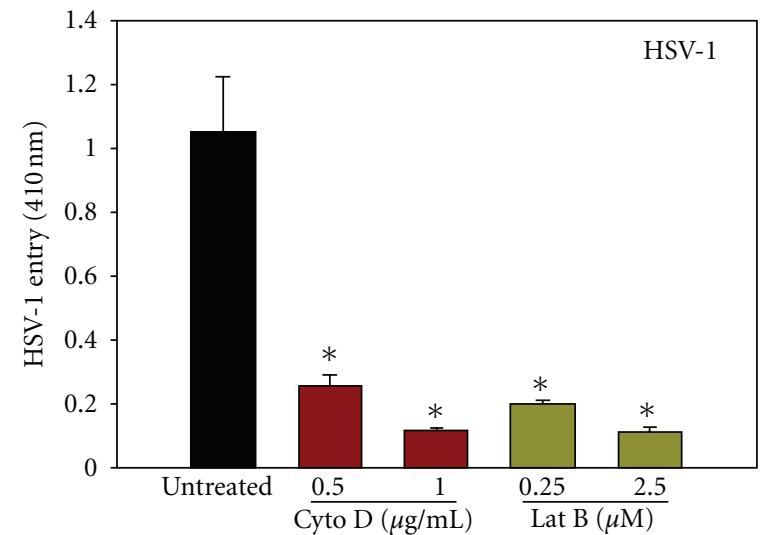

(d)

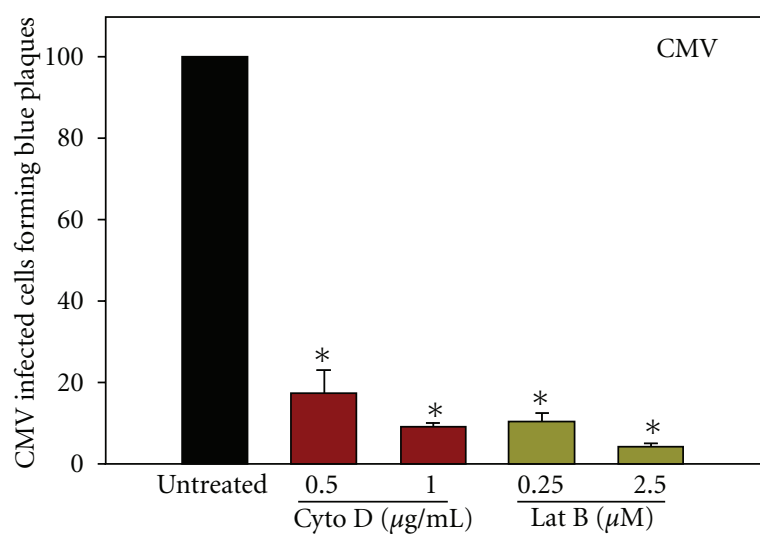

(e)

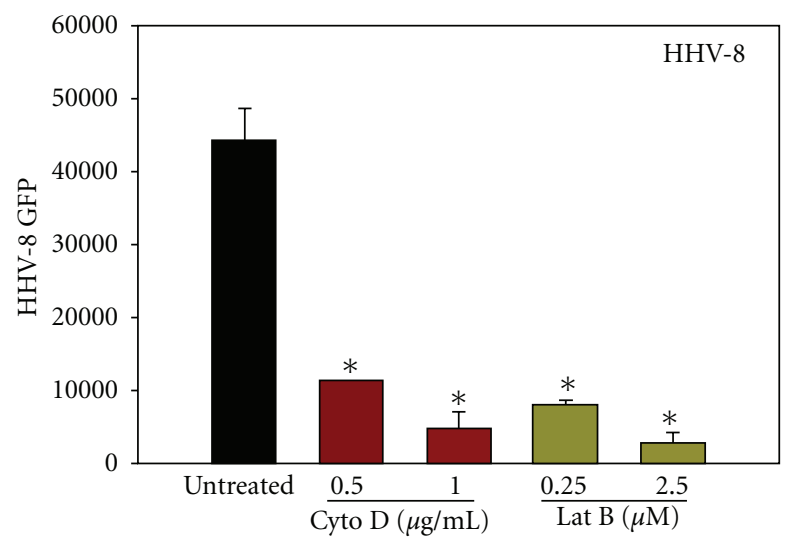

(f)

FIGURE 2: Herpesviruses can be internalized by membrane protrusions and blocking of protrusion formation blocks herpesvirus entry. (a)(c) High-resolution transmission electron micrographs (TEMs) of human ocular cells infected with herpesviruses are shown. Arrowheads in the panels indicate the association of viruses to cellular projections. (d)-(f) F-actin-dependent uptake of herpesviruses into ocular cells. Effects of actin depolymerizers (cytochalasin D; Cyto D and latrunculin b; Lat B) on virus entry into ocular cells (CF (d) for HSV-1, RPE cells for CMV (e), and HCjE for HHV-8 (f)) were examined. Cells were treated with Cyto D or Lat B at concentrations indicated for 90 min before exposure to the virus indicated. Asterisks indicate significant differences from controls $(P<0.05, t$-test $n=14$, error bars represent $\mathrm{SD})$. 


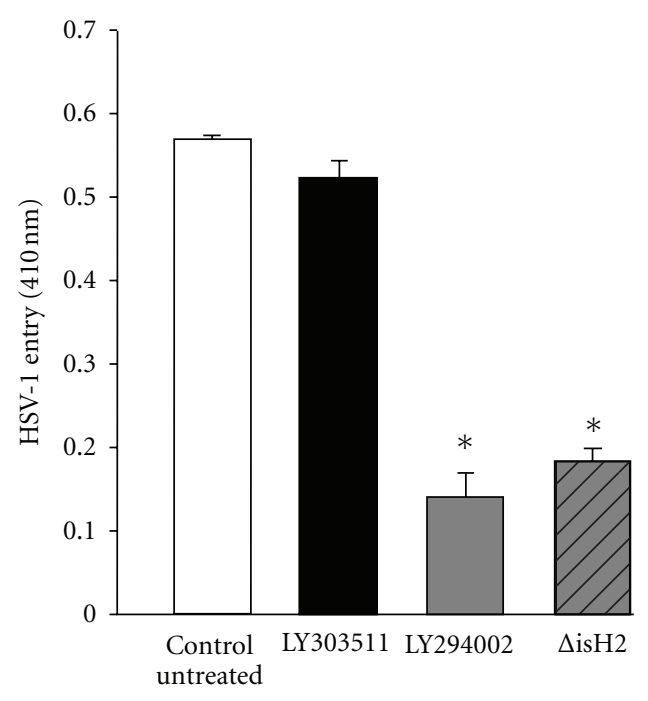

(a)

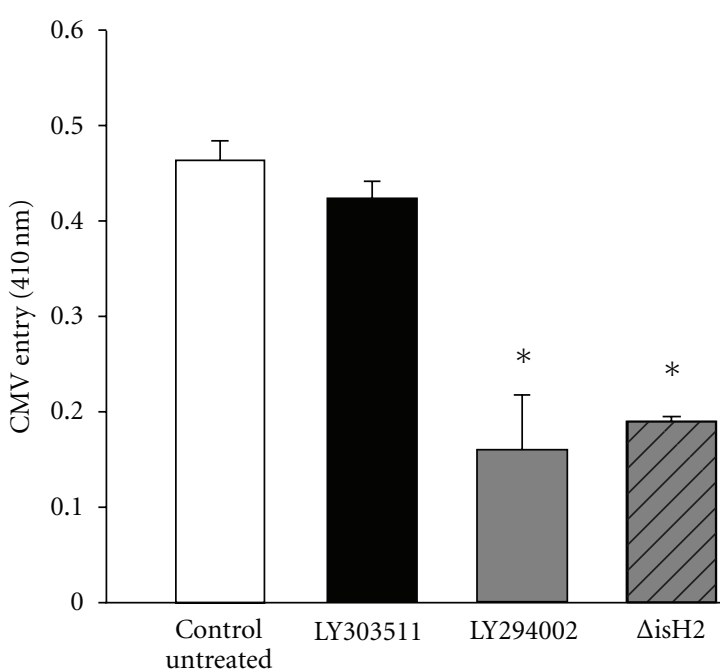

(b)

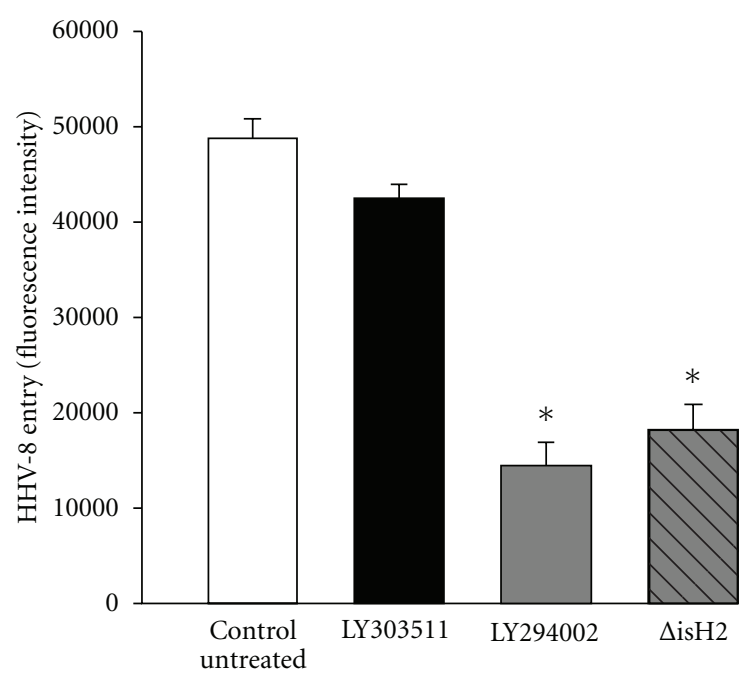

(c)

FIGURE 3: (a)-(c) Inhibition of PI3 Kinase signaling pathway negatively affects herpesvirus entry into cell. Cells were either treated with the compound indicated or transfected with PI3-dominant negative plasmid ( $\triangle \mathrm{iSH} 2$ ) followed by infection with HSV-1 (a), CMV (b), and HHV-8 (c). Results from viral entry assay are shown.

phagocytosis for entry. Our observation is also supported by the fact that additional intracellular signaling that may be needed for protrusion formation, such as activation of Rho family of GTPases, has already been reported with many herpesviruses $[15,36]$. The most significant contribution of our study is to demonstrate the possibility that involvement of membrane protrusions may be common, and perhaps crucial, for herpesvirus infection of the ocular cell types. Herpesviruses tend to spread by asymptomatic shedding, which allows normal looking individuals to transmit viruses to healthy individuals [37]. In the absence of effective vaccines against many herpesviruses, new and effective wide spectrum prophylactics are desperately needed to prevent an alarming rate of increase in herpesvirus transmission. In this regard, our study identifies an important common target for future intervention against multiple herpesviruses.
It may be that not just herpes but many additional unrelated viruses also begin their journey to infection by interacting with membrane protrusions and exploiting phagocytosis or a very similar mechanism for infection. Our ultimate goal is to identify how the virions successfully exit from degradation in phagolysosomes.

\section{Acknowledgments}

This paper was supported by NIH RO1 Grants Al057860 (DS) and P30 EY01792 (core grant) and a Lew Wasserman Merit Award from Research to Prevent Blindness (D. Shukla). V. Tiwari was supported by a grant award from Illinois Society for Prevention to Blindness (ISPB). The authors thank Dr. Kahn C. Roland (Harvard Medical School, USA) for providing them the PI3K dominant-negative constructs. 
Human CF and human conjunctival epithelial cells were kindly provided by Dr. Beatrice Yue (Lions of Illinois Eye Research Institute, University of Illinois at Chicago, USA) and Dr. Ilene Gipson (Harvard Medical School, USA).

\section{References}

[1] R. S. Flannagan, V. Jaumouillé, and S. Grinstein, "The cell biology of phagocytosis," Annual Review of Pathology, vol. 7, pp. 61-98, 2012.

[2] J. A. Swanson, M. T. Johnson, K. Beningo, P. Post, M. Mooseker, and N. Araki, "A contractile activity that closes phagosomes in macrophages," Journal of Cell Science, vol. 112, part 3, pp. 307-316, 1999.

[3] Q. Lu, Q. Li, and Q. Lu, "Regulation of phagocytosis by TAM receptors and their ligands," Frontiers of Biology in China, vol. 5, no. 3, pp. 227-237, 2010.

[4] C. Clement, V. Tiwari, P. M. Scanlan, T. Valyi-Nagy, B. Y. J. T. Yue, and D. Shukla, "A novel role for phagocytosis-like uptake in herpes simplex virus entry," Journal of Cell Biology, vol. 174, no. 7, pp. 1009-1021, 2006.

[5] E. Ghigo, J. Kartenbeck, P. Lien et al., "Ameobal pathogen mimivirus infects macrophages through phagocytosis," PLoS Pathogens, vol. 4, no. 6, Article ID e1000087, 2008.

[6] E. Ghigo, "A dilemma for viruses and giant viruses: which endocytic pathway to use to enter cells?" Intervirology, vol. 53, no. 5, pp. 274-283, 2010.

[7] R. C. May and L. M. Machesky, "Phagocytosis and the actin cytoskeleton," Journal of Cell Science, vol. 114, part 6, pp. 10611077, 2001.

[8] F. Niedergang and P. Chavrier, "Signaling and membrane dynamics during phagocytosis: many roads lead to the phagos(R)ome," Current Opinion in Cell Biology, vol. 16, no. 4, pp. 422-428, 2004.

[9] D. Shukla and P. G. Spear, "Herpesviruses and heparan sulfate: an intimate relationship in aid of viral entry," Journal of Clinical Investigation, vol. 108, no. 4, pp. 503-510, 2001.

[10] G. N. Holland, "AIDS and ophthalmology: the first quarter century," American Journal of Ophthalmology, vol. 145, no. 3, pp. 397-408, 2008.

[11] J. Thomas and B. T. Rouse, "Immunopathogenesis of herpetic ocular disease," Immunologic Research, vol. 16, no. 4, pp. 375386, 1997.

[12] H. Minoda, N. Usui, T. Sata, H. Katano, H. Serizawa, and S. Okada, "Human herpesvirus-8 in Kaposi's sarcoma of the conjunctiva in a patient with AIDS," Japanese Journal of Ophthalmology, vol. 50, no. 1, pp. 7-11, 2006.

[13] N. Miller and L. M. Hutt-Fletcher, "Epstein-Barr virus enters B cells and epithelial cells by different routes," Journal of Virology, vol. 66, no. 6, pp. 3409-3414, 1992.

[14] A. V. Nicola, A. M. McEvoy, and S. E. Straus, "Roles for endocytosis and low $\mathrm{pH}$ in herpes simplex virus entry into HeLa and Chinese hamster ovary cells," Journal of Virology, vol. 77, no. 9, pp. 5324-5332, 2003.

[15] N. Sharma-Walia, P. P. Naranatt, H. H. Krishnan, L. Zeng, and B. Chandran, "Kaposi's sarcoma-associated herpesvirus/human herpesvirus 8 envelope glycoprotein $\mathrm{gB}$ induces the integrin-dependent focal adhesion kinase-srcphosphatidylinositol 3-kinase-rho GTPase signal pathways and cytoskeletal rearrangements," Journal of Virology, vol. 78, no. 8, pp. 4207-4223, 2004.

[16] P. G. Spear and R. Longnecker, "Herpesvirus entry: an update," Journal of Virology, vol. 77, no. 19, pp. 10179-10185, 2003.
[17] A. V. Nicola and S. E. Straus, "Cellular and viral requirements for rapid endocytic entry of herpes simplex virus," Journal of Virology, vol. 78, no. 14, pp. 7508-7517, 2004.

[18] R. S. B. Milne, A. V. Nicola, J. C. Whitbeck, R. J. Eisenberg, and G. H. Cohen, "Glycoprotein D receptor-dependent, low$\mathrm{pH}$-independent endocytic entry of herpes simplex virus type 1," Journal of Virology, vol. 79, no. 11, pp. 6655-6663, 2005.

[19] A. V. Nicola, J. Hou, E. O. Major, and S. E. Straus, "Herpes simplex virus type 1 enters human epidermal keratinocytes, but not neurons, via a $\mathrm{pH}$-dependent endocytic pathway," Journal of Virology, vol. 79, no. 12, pp. 7609-7616, 2005.

[20] J. Hori, J. L. Vega, and S. Masli, "Review of ocular immune privilege in the year 2010: modifying the immune privilege of the eye," Ocular Immunology and Inflammation, vol. 18, no. 5, pp. 325-333, 2010.

[21] V. Tiwari, M. J. Oh, M. Kovacs, S. Y. Shukla, and D. Shukla, "Role for nectin-1 in herpes simplex virus 1 entry and spread in human retinal pigment epithelial cells," FEBS Journal, vol. 275, no. 21, pp. 5272-5285, 2008.

[22] V. Tiwari, C. Clement, D. Xu et al., "Role for 3-O-sulfated heparan sulfate as the receptor for herpes simplex virus type 1 entry into primary human corneal fibroblasts," Journal of Virology, vol. 80, no. 18, pp. 8970-8980, 2006.

[23] J. Akhtar, V. Tiwari, M.-J. Oh et al., "HVEM and nectin1 are the major mediators of herpes simplex virus (HSV1) entry into human conjuctival epithelium," Investigative Ophthalmology \& Visual Science, vol. 49, pp. 4026-4035, 2008.

[24] V. Tiwari and D. Shukla, "Phosphoinositide 3 kinase signalling may affect multiple steps during herpes simplex virus type-1 entry," Journal of General Virology, vol. 91, no. 12, pp. 30023009, 2010.

[25] I. Spector, N. R. Shochet, D. Blasberger, and Y. Kashman, "Latrunculins-novel marine macrolides that disrupt mocrofilament organization and affect cell growth: comparison with cytochalasin D," Cell Motility and the Cytoskeleton, vol. 13, pp. 127-144, 1989.

[26] M. J. Lehmann, N. M. Sherer, C. B. Marks, M. Pypaert, and W. Mothes, "Actin- and myosin-driven movement of viruses along filopodia precedes their entry into cells," Journal of Cell Biology, vol. 170, no. 2, pp. 317-325, 2005.

[27] D. Shukla, J. Liu, P. Blaiklock et al., "A novel role for 3-Osulfated heparan sulfate in herpes simplex virus 1 entry," Cell, vol. 99, no. 1, pp. 13-22, 1999.

[28] W. G. King, M. D. Mattaliano, T. O. Chan, P. N. Tsichlis, and J. S. Brugge, "Phosphatidylinositol 3-kinase is required for integrin-stimulated AKT and Raf-1/mitogen-activated protein kinase pathway activation," Molecular and Cellular Biology, vol. 17, no. 8, pp. 4406-4418, 1997.

[29] S. Wennström and J. Downward, "Role of phosphoinositide 3-kinase in activation of Ras and mitogen- activated protein kinase by epidermal growth factor," Molecular and Cellular Biology, vol. 19, no. 6, pp. 4279-4288, 1999.

[30] K. Ueki, P. Algenstaedt, F. Mauvais-Jarvis, and C. R. Kahn, "Positive and negative regulation of phosphoinositide 3kinase-dependent signaling pathways by three different gene products of the $\mathrm{p} 85 \alpha$ regulatory subunit," Molecular and Cellular Biology, vol. 20, no. 21, pp. 8035-8046, 2000.

[31] C. L. Carpenter, K. R. Auger, M. Chanudhuri et al., "Phosphoinositide 3-kinase is activated by phosphopeptides that bind to the SH2 domains of the $85-\mathrm{kDa}$ subunit," Journal of Biological Chemistry, vol. 268, no. 13, pp. 9478-9483, 1993.

[32] I. D. Hiles, O. Masayuki, S. Volinia et al., "Phosphatidylinositol 3-kinase: structure and expression of the $110 \mathrm{kd}$ catalytic subunit," Cell, vol. 70, no. 3, pp. 419-429, 1992. 
[33] E. Y. Skolnik, B. Margolis, M. Mohammadi et al., "Cloning of PI3 kinase-associated p85 utilizing a novel method for expression/cloning of target proteins for receptor tyrosine kinases," Cell, vol. 65, no. 1, pp. 83-90, 1991.

[34] B. Stoyanov, S. Volinia, T. Hanck et al., "Cloning and characterization of a $\mathrm{G}$ protein-activated human phosphoinositide-3 kinase," Science, vol. 269, no. 5224, pp. 690-693, 1995.

[35] P. J. Coffer, N. Geijsen, L. M'rabet et al., "Comparison of the roles of mitogen-activated protein kinase kinase and phosphatidylinositol 3-kinase signal transduction in neutrophil effector function," Biochemical Journal, vol. 329, part 1, pp. 121-130, 1998.

[36] N. Cheshenko, W. Liu, L. M. Satlin, and B. C. Herold, "Focal adhesion kinase plays a pivotal role in herpes simplex virus entry," Journal of Biological Chemistry, vol. 280, no. 35, pp. 31116-31125, 2005.

[37] H. E. Kaufman, A. M. Azcuy, E. D. Varnell, G. D. Sloop, H. W. Thompson, and J. M. Hill, "HSV-1 DNA in tears and saliva of normal adults," Investigative Ophthalmology and Visual Science, vol. 46, no. 1, pp. 241-247, 2005. 


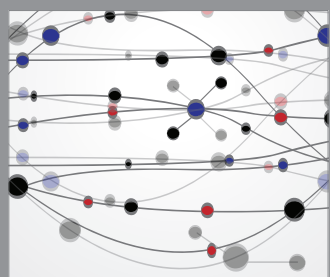

The Scientific World Journal
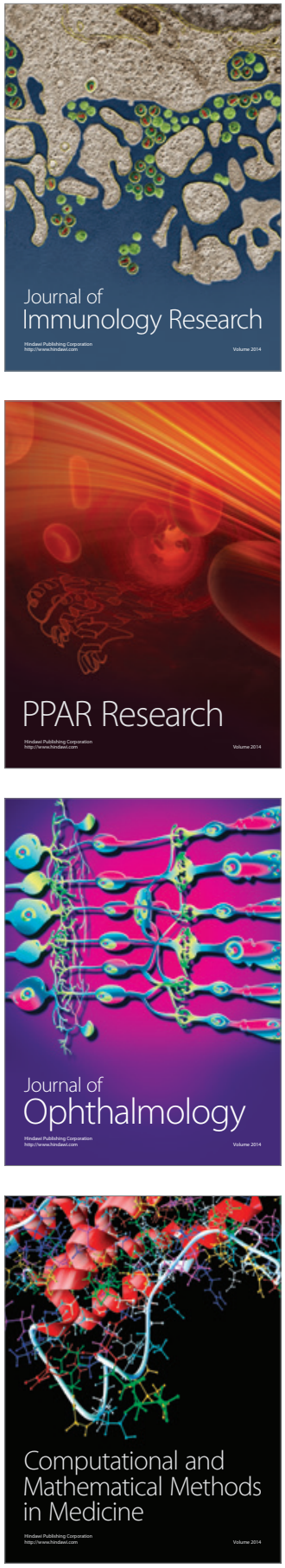

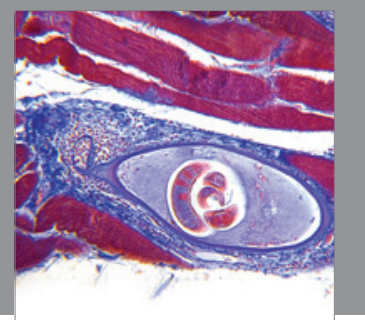

Gastroenterology

Research and Practice
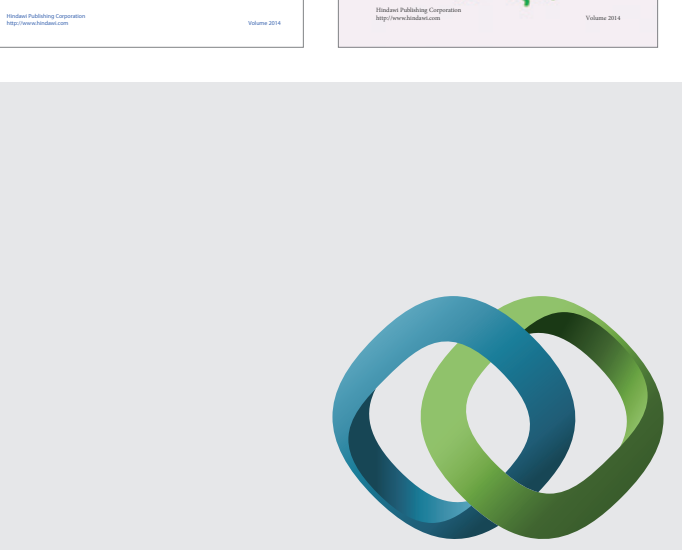

\section{Hindawi}

Submit your manuscripts at

http://www.hindawi.com
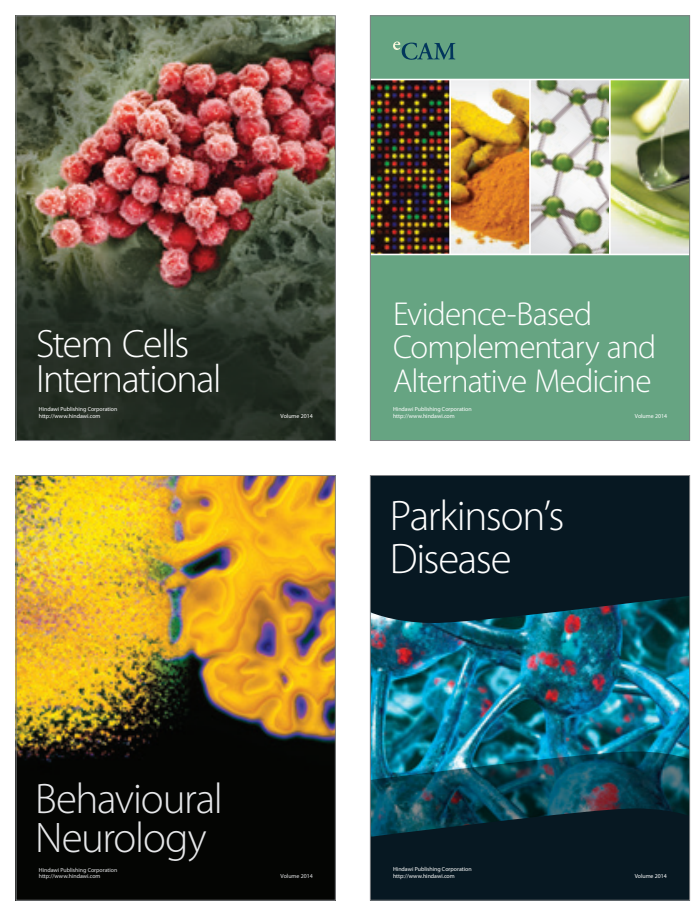

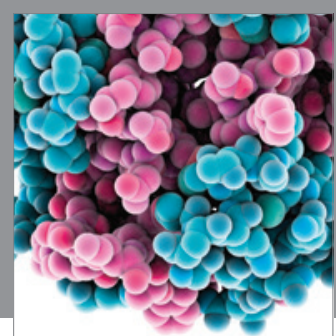

Journal of
Diabetes Research

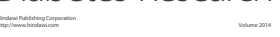

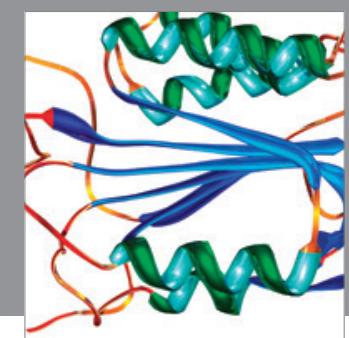

Disease Markers
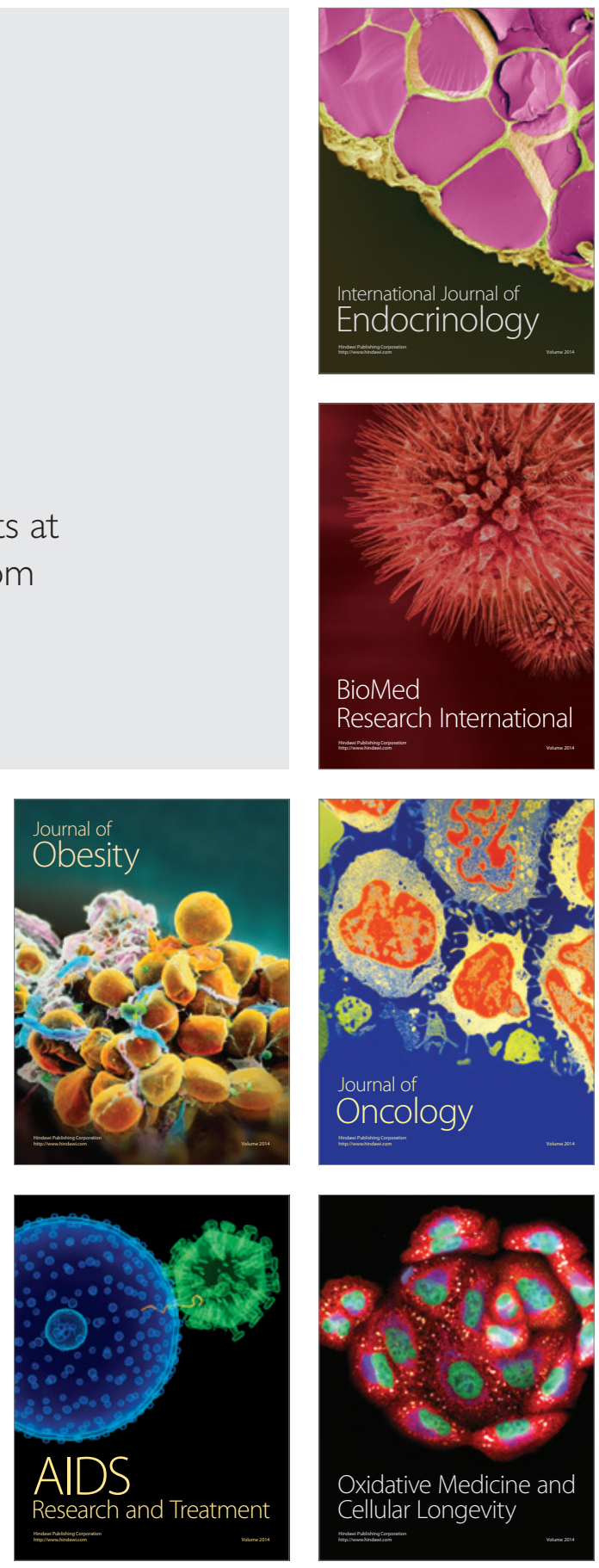